\title{
Optical and THz Galois diffusers
}

M. Jaax

\section{S. Wolff}

B. Laegel

\section{H. Fouckhardt}

fouckhar@physik.uni-kl.de
Physics Department, Integrated Optoelectronics and Microoptics Research Group, Kaiserslautern University of Technology, P.O. Box 3049, 67653 Kaiserslautern, Germany

Nano Structuring Center, Research Center OPTIMAS, Kaiserslautern University of Technology, P.O. Box 3049, 67653 Kaiserslautern, Germany

Nano Structuring Center, Research Center OPTIMAS, Kaiserslautern University of Technology, P.O. Box 3049, 67653 Kaiserslautern, Germany

Physics Department, Integrated Optoelectronics and Microoptics Research Group, Kaiserslautern University of Technology, P.O. Box 3049, 67653 Kaiserslautern, Germany

Binary surface reliefs with sub-wavelength features making up a pseudorandom pattern based on mathematical Calois fields $\mathrm{CF}\left(\mathrm{p}^{m}\right)[1,2]$ can scatter incoming waves into a large number of diffraction maxima within a huge solid angle. A one-dimensional (1D) Galois number sequence can be folded into a two-dimensional (2D) array by the sino-representation [2]. This concept was been verified for acoustic waves a long time ago $[3,4]$ and is investigated here for visible light and $\mathrm{THz}$ waves. Our Galois diffusers are designed as reflection reliefs and realised by electron beam lithography for the optical regime and UV photolithography for the THz regime. Our results show that optical and $\mathrm{THz}$ Galois surfaces are excellent diffusers for electromagnetic waves; they distribute the reflected intensity evenly over a large number of maxima nearly within the entire half solid angle in the backward direction.

[DOI: http://dx.doi.org/10.2971/jeos.2013.13020]

Keywords: Diffractive optical elements, sub-wavelength structures, THz, diffuser, Galois fields

\section{INTRODUCTION}

For the realisation of optical diffusers by structuring the surface two different approaches are possible: the surfaces can be roughened or diffractive optical elements (DOEs) can be created on the surfaces. For roughened surfaces the fraction of diffuse to specular scattered light intensity can be chosen by an appropriate selection of the roughness morphology [5]. These diffusers scatter the light monotonically over a very large scattering angle (up to $\approx 140^{\circ}$ ). DOEs that are used as diffusers [6], beam splitters $[7,8]$ or for flat-top beamshaping applications $[9,10]$ are well established. They can be used to distribute the intensity uniformly over a given number of diffraction orders. In order to show that DOEs with features on a sub-wavelength scale can scatter incoming waves over a large number of maxima within a huge scattering angle $\left(\approx 180^{\circ}\right)$, we have chosen an approach well known from acoustics, so called Galois diffusers. They represent a special kind of Dammann gratings [11, 12], designed as binary reflection gratings, where the surface reliefs represent pseudorandom sequences based on mathematical Galois fields $\operatorname{GF}\left(\mathrm{p}^{m}\right)$ $[1,2]$. The binary Galois maximum length sequences $\operatorname{GF}\left(2^{m}\right)$ consist of bit strings, which are repeated until the maximum number of permutations is reached. The one-dimensional (1D) bit sequence can be mapped onto a two-dimensional (2D) array by the so-called sino-representation [2]. For applications, such as reflective scattering plates, the individual bits have a lateral width of half the wavelength (i.e. $\lambda / 2)$ and a height of a quarter wavelength $(\lambda / 4)$ or 0 , as depicted in Figure 1.

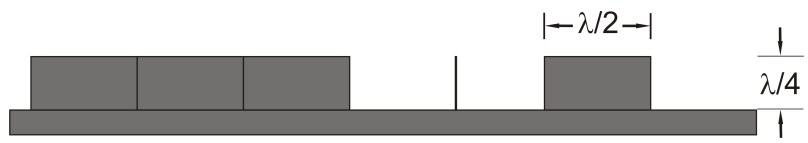

FIG. 1 Sketch of a 1 D reflective Galois diffuser $\mathrm{GF}\left(2^{3}\right)$.

Theoretically, an incoming wave should be uniformly scattered over a large number of diffraction maxima within a large solid angle, while the specular peak is significantly suppressed by such a plate [2,3]. Acoustical Galois diffusers are easy to prepare, because width and height of the individual scattering cuboid elements are of the order of several centimeters. To apply this principle to the scattering of electromagnetic waves the diffusers should be made from a highly conductive material, which is the analogue to the hard materials used in the acoustical case [13]. However, the technological challenge is the preparation of cuboid structures in the $\mu \mathrm{m}$ - or nm-range with aspect ratios of $\geq 0.5$. The numerical simulations of Galois diffusers show that it is essential for the functionality that the manufacturing tolerance is kept below $2 \%$. We have realised binary Galois diffusers for both the $\mathrm{THz}$ and the visible wavelength regime. We demonstrate that for the $\mathrm{THz}$ frequency range various Galois diffusers of very good accuracy can be produced. Even for the optical wavelength regime we present sub-wavelength Galois-type DOEs which suppress the specular peak to nearly the same intensity as that 

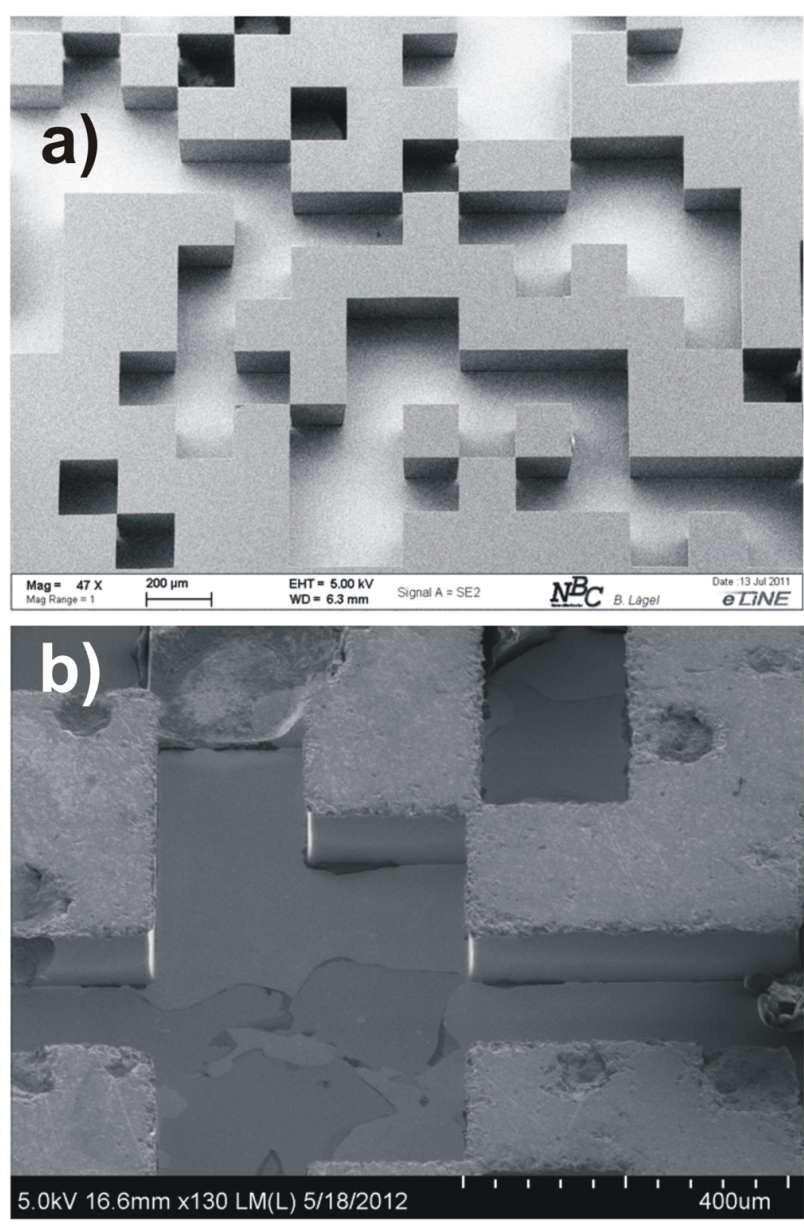

FIG. 2 a) Photolithographically patterned photoresist $\left(A Z^{\circledR} 125 \mathrm{nXT}\right)$ with a height of $120 \mu \mathrm{m}$; b) final sample after lapping, polishing, and stripping of the Ni plating at a height of $100 \mu \mathrm{m}$. In both images the lateral width of one cuboid is $200 \mu \mathrm{m}$

of the scattering/diffraction orders with a distribution over a planar scattering angle range of $140^{\circ}$.

\section{THZ REGIME}

The Galois diffusers were designed for a wavelength of 400 $\mu \mathrm{m}$ which corresponds to a frequency of $0.75 \mathrm{THz}$. Accordingly, the lateral width is $200 \mu \mathrm{m}$. The technological process of the samples for the $\mathrm{THz}$ regime consists of photolithography, electroplating, lapping, and polishing. In order to avoid a dominant specular peak, the sidewalls of the structures have to be vertical and the structure height has to be exactly $100 \mu \mathrm{m}$ $\pm 2 \%$. A silicon wafer is used as a substrate, which is coated by electron beam evaporation with a $200 \mathrm{~nm}$ thick Cu layer first. On top of this $\mathrm{Cu}$ layer a $120 \mu \mathrm{m}$ thick photoresist $\mathrm{AZ}{ }^{\circledR} 125$ nXT layer is spin-coated, and subsequently patterned by photolithography. The processed wafers are electroplated with Ni. The subsequent lapping and polishing steps remove the overgrown Ni until the Ni layer has a thickness of $100 \mu \mathrm{m}$. In a final step, the remaining photoresist is stripped.

Figure 3 shows the results of the frequency- and angleresolved measurements of the scattered/diffracted intensity (colour-coded). In reference [14] the THz pulse was focused by Si-lenses. However, for our measurements the lenses were removed. Therefore the beam waist of the $\mathrm{THz}$ pulse has

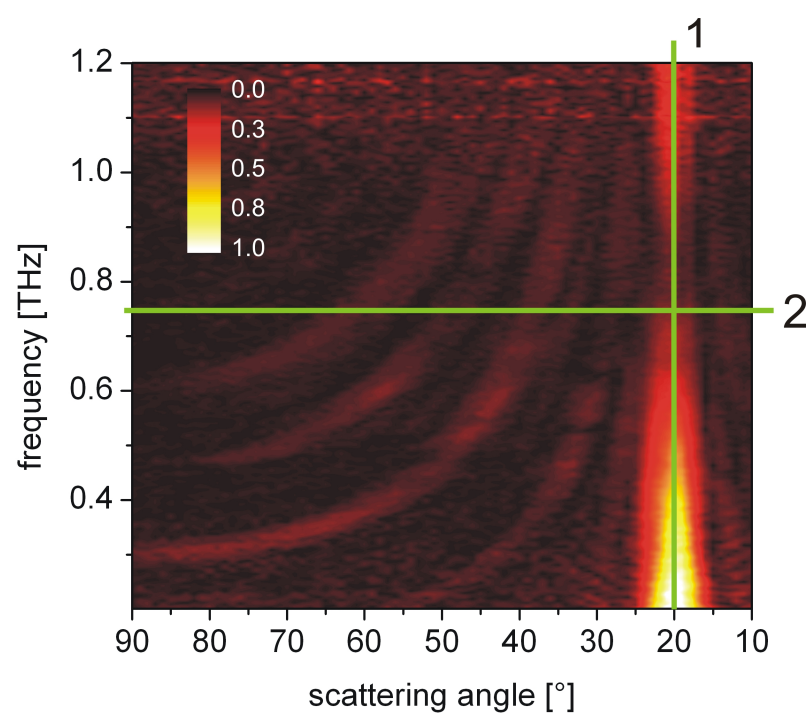

FIG. 3 Scattering results of a THz $2 \mathrm{D}$ Galois diffuser $\mathrm{GF}\left(2^{8}\right)$. The colour code in the legend is the normalised intensity.

a cross-section of about $2 \mathrm{~cm} \times 2 \mathrm{~cm}$, which corresponds approximately to the lateral width of the samples. In Figure 3 the frequency is plotted against the angle of detection, where the intensity is colour-coded. The highest intensity, normalised to 1 , corresponds to a very bright yellow tone. The angle of incidence is $20^{\circ}$, such that the specular peak occurs at $20^{\circ}$. The plot is generated from two measurements. First, as a reference, the scattering spectrum of a smooth metal plate is acquired. Then the scattering spectrum of a structured Galois surface is detected. Then the intensity values for the structured sample are normalised to the specular peak of the reference at $20^{\circ}$. Figure 3 shows the colour plot of the normalised scattering spectrum of a 2D Galois diffuser, based on an $8 \times 8$ array of a Galois sequence of $\mathrm{GF}\left(2^{8}\right)$. At the design frequency of $0.75 \mathrm{THz}$ four secondary maxima are expected, which can be seen in Figure 3 and in Figure 4(b).

Figure 4(a) (black line) corresponds to a line scan at $20^{\circ}$ in Figure 3 (line 1). To get an impression of the special scattering characteristics of a Galois diffuser, the scattering spectrum of a chequerboard pattern is added in both graphs for comparison. The chequerboard has the same design frequency as the Galois diffuser, which means, that the individual cuboids have a lateral width of $200 \mu \mathrm{m}$ and a height of $100 \mu \mathrm{m}$. The measured scattering characteristics are in good agreement with the expected ones. The graph of the angle resolved scattering characteristics of the Galois diffuser in Figure 4(b) corresponds to the second line scan, marked 2 in Figure 3. For comparison, the scattering spectrum of the chequerboard at the minimum at the frequency of $0.7 \mathrm{THz}$ is shown. It can be seen, that the scattering spectrum of the chequerboard has one large specular and two minor peaks at $12^{\circ}$ and $22^{\circ}$, respectively, while the Galois diffuser has four peaks, which are distributed over a large scattering angle range.

\section{OPTICAL REGIME}

For the realisation of optical Galois diffusers different approaches have been tested. We started with prototypes con- 
a) frequency-resolved

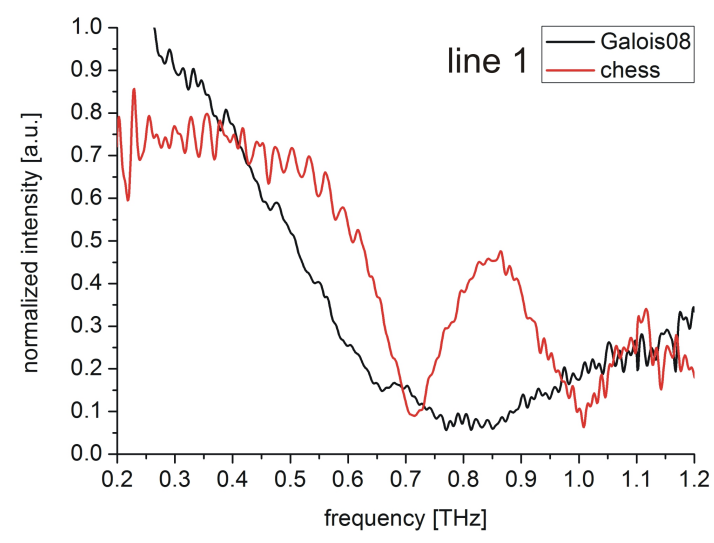

b) angle-resolved

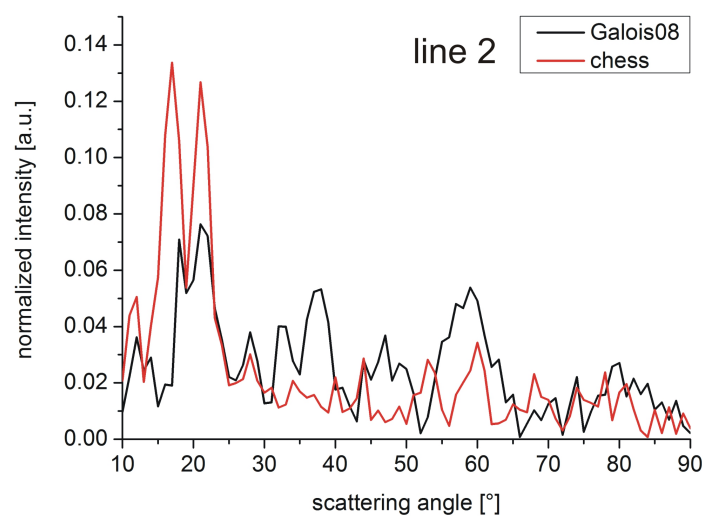

FIG. 4 Comparison of the scattered intensities on the lines indicated in Figure 3 of a $2 \mathrm{D}$ Galois diffuser $\operatorname{CF}\left(2^{8}\right)$ with a chequerboard pattern. The lines between the measured data points are guides to the eye.

sisting of a PMMA layer on a Si wafer only, such that the thickness of the PMMA generates the required phase delay. This relatively simple method enables quick tests of the scattering characteristics of the generated patterns, but the samples are fragile. Therefore, we also fabricated Galois diffusers by electron beam lithography and ICP-RIE dry-etching with a layer system consisting of $\mathrm{Cr}$ and $\mathrm{Ti}$ on a Si wafer, see Figure 5.

On top of the Si wafer three metallic layers of defined thickness are sputtered (110 nm Cr, $140 \mathrm{~nm} \mathrm{Ti,} 20 \mathrm{~nm} \mathrm{Cr}$ ). A $170 \mathrm{~nm}$ thick PMMA950K layer is spin-coated on top and patterned by electron beam lithography. In a first dry-etching process the first $\mathrm{Cr}$ layer is structured with a $\mathrm{Cl}_{2} / \mathrm{O}_{2}$ plasma. After the $20 \mathrm{~nm} \mathrm{Cr}$ layer has been etched through, the remaining resist is removed. In a second dry-etching process the $20 \mathrm{~nm} \mathrm{Cr}$ mask serves as etch mask for the underlying Ti layer. Using a $\mathrm{SF}_{6} / \mathrm{O}_{2}$ plasma the Ti layer is etched completely through to the bottom $\mathrm{Cr}$ layer which forms an etch-stop. The targeted thickness of the metal structure - in this case $160 \mathrm{~nm}$ - was met with a tolerance of $\pm 1 \mathrm{~nm}$. Figure 6 shows an SEM image of a finished sample.

Angle-resolved and polarisation-dependent light scattering measurements are performed under elimination of stray light in a shielded box. The sample is irradiated with a polarised and focused laser beam (642 nm) at an angle of $<2^{\circ}$ normal to the patterned sample surface. Figure 7 shows a sketch of

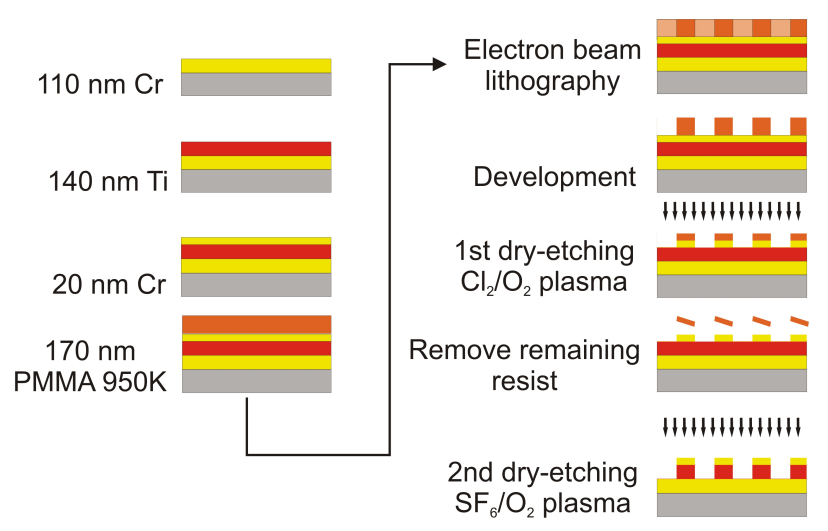

FIG. 5 Flow diagram for the process of an optical 2D scatterer.

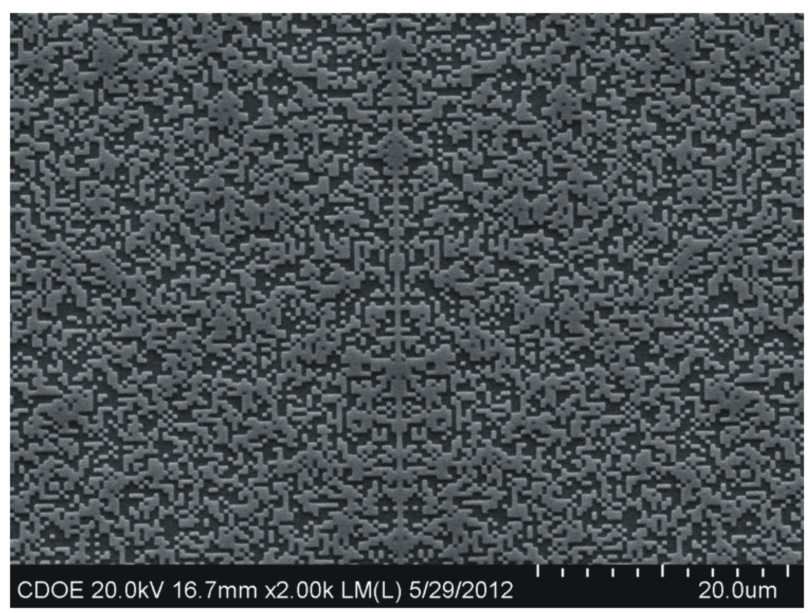

FIG. 6 Finished optical Galois diffuser, showing the structured layer system.

the optical measurement setup. The sample holder is located in the middle of the test setup, such that the detector, which is attached to a $50 \mathrm{~cm}$ long arm, can be moved in a plane around the sample. Due to the small non-zero angle of incidence the detector can be moved in the plane without crossing the laser beam. In the focal spot the laser beam has a diameter of $<1 \mathrm{~mm}$, such that only the structured surface of $(1.3 \times 1.3) \mathrm{mm}^{2}$ is illuminated. An unstructured surface area on the same sample is chosen as a reference. In the following results the polarisation is defined through the small angle of incidence, giving a plane of incidence.

Figure 8 gives the far-field intensity as a function of the scattering angle for two different optical Galois diffusers, see Figure 8(a) for a 1D scatterer and Figure 8(b) for a 2D scatterer. 1D means that the grating is based on lines which are arranged according to a Galois number sequence. 2D indicates that it is a Galois sequence that has been transferred into a 2D array by the sino representation [2]. The corresponding far-fields also show a one- or a two-dimensional structure, respectively. Accordingly, the result for the 1D scatterer, which is based on a Galois sequence $\mathrm{GF}\left(2^{4}\right)$, shows all $2^{4}-1=15$ existing diffraction orders. The $2 \mathrm{D}$ scatterer is based on a $\mathrm{GF}\left(2^{8}\right)$ (Figure 7 , photograph) which means, that 255 diffraction orders exist which are arranged in an $15 \times 17$ array. As an example a horizontal line scan is shown in Figure 8(b). As mentioned above, for each sample a separate reference measurement is carried out and the diffraction orders are normalised to the maximum of the specular peak of the reference. The 1D scatterer is a proto- 

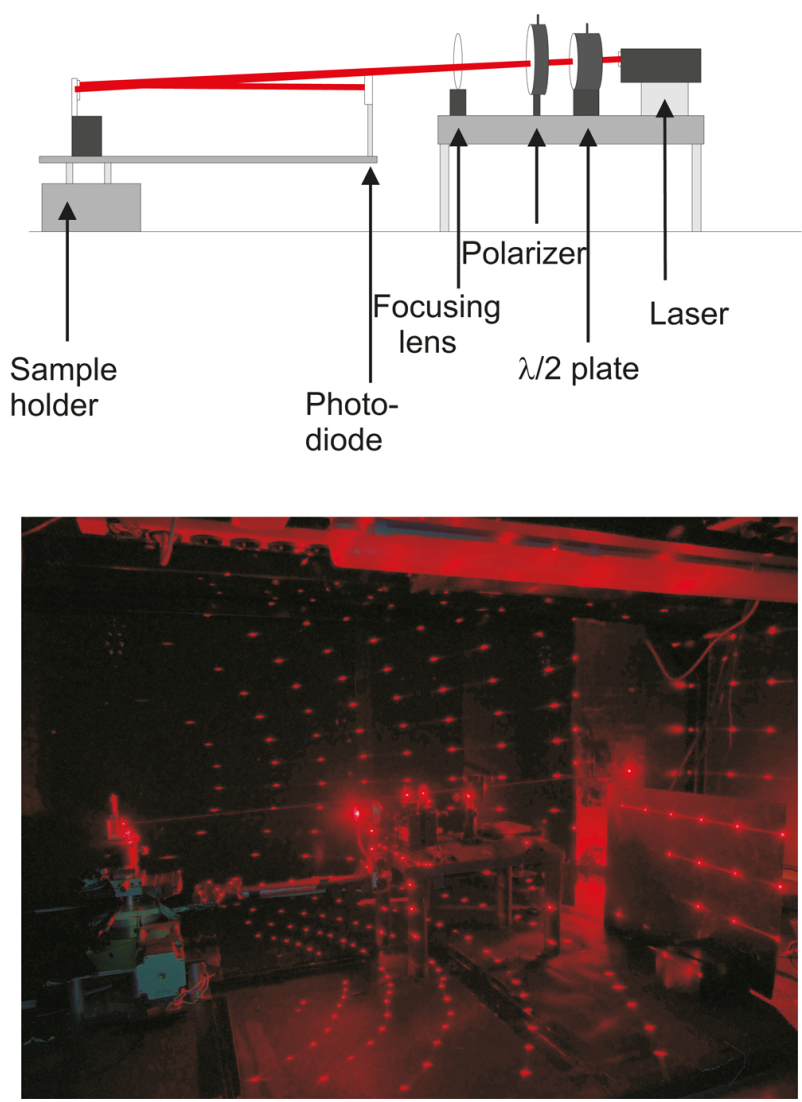

FIG. 7 Schematic diagram and photograph of the scattered light measurement setup; the light pattern results from a $2 \mathrm{D}$ Galois diffuser $\mathrm{GF}\left(2^{8}\right)$, exhibiting the desired multitude of diffraction maxima.

type, which means it is made of patterned PMMA on Si, while the $2 \mathrm{D}$ scatterer is a metallic sample, which is prepared by the method described above. The angle-resolved far-field of the illustrated optical Galois diffusers shows a similar behaviour as the Galois scatterer in the $\mathrm{THz}$ regime. In the $1 \mathrm{D}$ case all orders have nearly the same intensity. In the $2 \mathrm{D}$ case, the intensity is distributed evenly over all orders in the entire hemisphere of reflection (see photograph in Figure 7). Only the specular peak, i.e. the zeroth order, has an about an order of magnitude higher peak intensity than the other orders. Nonetheless the energy within the zeroth order is small (about 4\%).

We are also able to change the polarisation of the laser beam, an option which is instructive in the case of sub-wavelength structures $[15,16]$. The measured optical scattering characteristics are recorded with a polarisation parallel to the plane of incidence. The polarisation has a strong influence on the 1D scatterer while the influence on the 2D scatterer is only measurable for designs based on very short Galois sequences, e.g. for $\mathrm{GF}\left(2^{4}\right)$. This effect is not surprising, because 2D Galois designs have a nearly equal distribution of zeros and ones in both directions. In the 1D case there is a nearly equal distribution in only one direction.

\section{CONCLUSION}

For the first time Galois diffusers have been realised for $\mathrm{THz}$ frequencies and the optical wavelength regime. We have shown that sub-wavelength structures arranged according

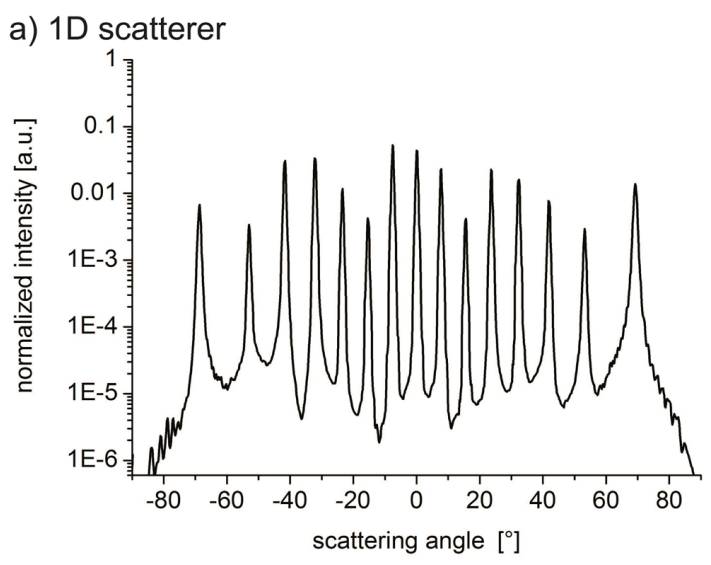

b) 2D scatterer

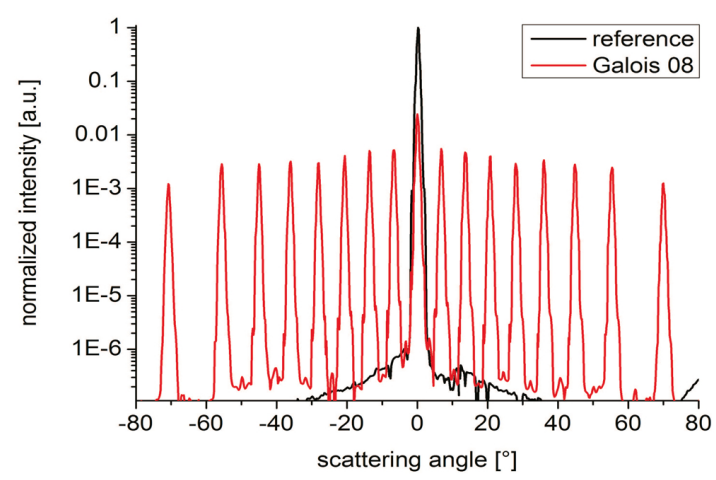

FIG. 8 Measured scattering characteristics of optical Galois diffusers:

a) $1 D$ scatterer $\operatorname{CF}\left(2^{4}\right)$ : The intensity is scattered into 15 orders, which are all arranged within one dimension. The specular peak is strongly suppressed. All orders are in the same intensity range;

b) $2 D$ scatterer $\operatorname{CF}\left(2^{8}\right)$ : The intensity is distributed over 255 orders. Only the specular peak (the zeroth order) carries slightly more energy than the remaining peaks (the other orders).

to Galois sequences scatter incoming waves evenly over a large number of maxima within a huge solid angle. Furthermore, Galois diffusers show a broad frequency-band scattering/diffraction behaviour around the frequency they have been designed for.

\section{ACKNOWLEDGEMENTS}

The authors acknowledge financial support by the German Research Foundation ('Deutsche Forschungsgemeinschaft', DFG) under contract Fo157/39. Help by 'Forschungsinstitut fuer mineralische und metallische Werkstoffe Edelsteine/Edelmetalle' (FEE), Idar-Oberstein, Germany (lapping and polishing), by Daniel Molter, TeraTec group of Fraunhofer IPM, Germany (THz measurments), by Simon Ediger and Monika Saumer, Univ. of Appl. Sc. Kaiserslautern/Zweibruecken, Germany (electroplating), by Stefan Trellenkamp and Juergen Moers, FZ Juelich, Germany (patterning and etching of 2D scatterer) is also gratefully acknowledged. 


\section{References}

[1] E. Bach, and J. Shallit, Algorithmic number theory (MIT Press, Cambridge, 1996).

[2] M. R. Schroeder, Number theory in science and communication (Springer, Berlin, 2006).

[3] B. Costa, A. Folacci, and P. Gabrielli, "Diffraction of ultrasonic waves from elastic Galois gratings," J. Appl. Phys. 79, 2879-2889 (1996).

[4] T. J. Cox, P. D'Antonio, and M. Schroeder, "Acoustic absorbers and diffusers, theory, design and application," J. Acoust. Soc. Am. 117, 289-330 (2005).

[5] H. Fouckhardt, E. Hein, D. Fox, and M. Jaax, "Multitude of glass surface roughness morphologies as a tool box for dosed optical scattering," Appl. Optics 49, 1364-1372 (2010).

[6] H. P. Herzig, and P. Kipfer, "Aperture-Modulated Diffusers (AMDs)," in International Trends in Optics and Photonics ICO IV, Asakura, ed., 247-257 (Springer, Berlin/Heidelberg, 1999).

[7] H. Dammann, "Optische Gitter - moderne Anwendungen," Physik in unserer Zeit 11, 83-91 (1980).

[8] H. P. Herzig, D. Prongué, and R. Daendliker, "Design and Fabrication of Highly Efficient Fan-Out Elements," Jpn. J. Appl. Phys. 29, L1307-L1309 (1990).

[9] T. R. Sales, "Efficient and uniform illumination with microlensbased band-limited diffusers," Photon. Spectra 44, 49-53 (2010).
[10] R. Bitterli, T. Scharf, H. P. Herzig, W. Noell, N. de Rooij, A. Bich, S. Roth, et al., "Fabrication and characterization of linear diffusers based on concave micro lens arrays," 0pt. Express 18, 14251-14261 (2010).

[11] H. Dammann, and K. Groertler, "High-efficiency in-line multiple imaging by means of multiple phase holograms," Opt. Commun. 3, 312-315 (1971).

[12] H. Dammann, and E. Klotz, "Coherent optical generation and inspection of two-dimensional periodic structures," Opt. Acta 24, 505-515 (1977).

[13] W. Petirsch, and A. Schwab, "Investigation of the field uniformity of a mode-stirred chamber using diffusers based on acoustic theory," IEEE Conf. Publ.: Electromagnetic Compatibility 41, 446-451 (1999).

[14] F. Ellrich, T. Weinland, D. Molter, J. Jonuscheit, and R. Beigang, "Compact fiber-coupled terahertz spectroscopy system pumped at $800 \mathrm{~nm}$ wavelength," Rev. Sci. Instrum. 82, 053102 (2011).

[15] M. E. Motamedi, "Micro-opto-electro-mechanical systems," Opt. Eng. 33, 3505-3517 (1994).

[16] W. Freese, T. Kaempfe, E.-B. Kley, and A. Tuennermann, "Design of binary subwavelength multiphase level computer generated holograms," Opt. Lett. 35, 676-678 (2010). 